Commun.Fac.Sci.Univ.Ank.Series A 1

Volume 62, Number 2, Pages 37-51 (2013)

ISSN $1303-5991$

\title{
ON LORENTZIAN TRANS-SASAKIAN MANIFOLDS
}

\author{
U.C. DE AND KRISHNENDU DE
}

\begin{abstract}
The object of the present paper is to study the Trans-Sasakian structure on a manifold with Lorentzian metric. Several interesting results are obtained on the manifold. Also conformally flat Lorentzian Trans-Sasakian manifolds have been studied. Next, in three- dimensional Lorentzian TransSasakian manifolds, explicit formulae for Ricci operator, Ricci tensor and curvature tensor are obtained. Also it is proved that a three-dimensional Lorentzian Trans-Sasakian manifold of type $(\alpha, \beta)$ is locally $\phi$ - symmetric if and only if the scalar curvature $r$ is constant provided $\alpha$ and $\beta$ are constants. Finally, we give some examples of three-dimensional Lorentzian Trans-Sasakian manifold.
\end{abstract}

\section{INTRODUCTION}

Let $M$ be an odd dimensional manifold with Riemannian metric $g$. It is well known that an almost contact metric structure $(\phi, \xi, \eta)$ (with respect to $g$ ) can be defined on $M$ by a tensor field $\phi$ of type $(1,1)$, a vector field $\xi$ and a 1 - form $\eta$. If $M$ has a Sasakian structure (Kenmotsu structure), then $M$ is called a Sasakian manifold (Kenmotsu manifold). Sasakian manifolds and Kenmotsu manifolds have been studied by several authors.

In the classification of Gray and Hervella [8] of almost Hermitian manifolds there appears a class, $W_{4}$, of Hermitian manifolds which are closely related to locally conformally Kaehler manifolds. An almost contact metric structure $(\phi, \xi, \eta, g)$ on $M$ is Trans-Sasakian [17] if $(M \times R, J, G)$ belongs to the class $W_{4}$, where $J$ is the almost complex structure on $M \times \mathrm{R}$ defined by

$$
J\left(X, f \frac{d}{d t}\right)=\left(\phi X-f \xi, \eta(X) \frac{d}{d t}\right)
$$

for all vector fields $X$ on $M, f$ is a smooth function on $M \times \mathbb{R}$ and $G$ is the product metric on $M \times \mathbb{R}$. This may be expressed by the condition [2]

$$
\left(\nabla_{X} \phi\right) Y=\alpha(g(X, Y) \xi-\eta(Y) X)+\beta(g(\phi X, Y) \xi-\eta(Y) \phi X)
$$

Received by the editors Jan 25, 2013, Accepted: Nov. 30, 2013.

2000 Mathematics Subject Classification. : 53C15, 53C25.

Key words and phrases. Lorentzian Trans-Sasakian manifold, $\eta$-Einstein manifold, conformally flat manifold, locally $\phi$-symmetric manifold. 
for smooth functions $\alpha$ and $\beta$ on $M$. Hence we say that the Trans-Sasakian structure is of type $(\alpha, \beta)$. In particular, it is normal and it generalizes both $\alpha$-Sasakian and $\beta$-Kenmotsu structures. From the formula (1.1) one easily obtains

$$
\begin{gathered}
\nabla_{X} \xi=-\alpha(\phi X)+\beta(X-\eta(X) \xi) . \\
\left(\nabla_{X} \eta\right) Y=-\alpha g(\phi X, Y)+\beta g(\phi X, \phi Y) .
\end{gathered}
$$

In 1981, Janssens and Vanhecke introduced the notion of $\alpha$ - Sasakian and $\beta$ Kenmotsu manifolds where $\alpha$ and $\beta$ are non zero real numbers. It is known that [6] Trans-Sasakian structures of type $(0,0),(0, \beta)$ and $(\alpha, 0)$ are cosymplectic ([1], $[2]), \beta$ - Kenmotsu ([6]) and $\alpha$-Sasakian ([6]) respectively. The local structure of Trans-Sasakian manifolds of dimension $n \geq 5$ has been completely characterized by Marrero [10]. He proved that a Trans-Sasakian manifold of dimension $n \geq 5$ is either cosymplectic or $\alpha$-Sasakian or $\beta$-Kenmotsu manifold. Trans-Sasakian manifolds have been studied by several authors ([3], [4], [5],[11], [18]).

Let $(x, y, z)$ be cartesian co-ordinates in $\mathbb{R}^{3}$, then $(\phi, \xi, \eta, g)$ given by

$$
\begin{gathered}
\xi=\frac{\partial}{\partial z}, \eta=d z-y d x \\
\phi=\left(\begin{array}{ccc}
0 & -1 & 0 \\
1 & 0 & 0 \\
0 & -y & 0
\end{array}\right), \quad g=\left(\begin{array}{ccc}
e^{z}+y^{2} & 0 & -y \\
0 & e^{z} & 0 \\
-y & 0 & 1
\end{array}\right)
\end{gathered}
$$

is a Trans-Sasakian structure of type $\left(\frac{-1}{2 e^{z}}, \frac{1}{2}\right)$ in $\mathbb{R}^{3}[2]$. In general, in a threedimensional $K$-contact manifold with structure tensors $(\phi, \xi, \eta, g)$ for a non-constant function $f$, if we define $\tilde{g}=f g+(1-f) \eta \otimes \eta$; then $(\phi, \xi, \eta, \tilde{g})$ is a Trans-Sasakian structure of type $\left(\frac{1}{f}, \frac{1}{2} \xi(\ln f)\right)[10]$.

Let $M$ be a differentiable manifold. When $M$ has a Lorentzian metric $g$, that is, a symmetric non degenerate $(0,2)$ tensor field of index 1 , then $M$ is called a Lorentzian manifold. Since the Lorentzian metric is of index 1, Lorentzian manifold $M$ has not only spacelike vector fields but also timelike and lightlike vector fields. This difference with the Riemannian case give interesting properties on the Lorentzian manifold. A differentiable manifold $M$ has a Lorentzian metric if and only if $M$ has a 1- dimensional distribution. Hence odd dimensional manifold is able to have a Lorentzian metric.

Therefore, it is very natural and interesting idea to define both a Trans-Sasakian structure and a Lorentzian metric on an odd dimensional manifold.

The paper is organized as follows. In Section 1, we give a brief account of Lorentzian Trans-Sasakian manifolds. After preliminaries, some basic results are given. In Section 4, we study conformally flat Lorentzian Trans-Sasakian manifolds. 
In the next section, explicit formulae for Ricci operator, Ricci tensor and curvature tensor are obtained for three-dimensional Trans-Sasakian manifolds. Also it is proved that a three-dimensional Lorentzian Trans-Sasakian manifold of type $(\alpha, \beta)$ is locally $\phi$ - symmetric if and only if the scalar curvature $r$ is constant provided $\alpha$ and $\beta$ are constants. Finally we construct some examples of three-dimensional Lorentzian Trans-Sasakian manifolds.

\section{LORENTZIAN TRANS-SASAKIAN MANIFOLDS}

A differentiable manifold $M$ of dimension $(2 n+1)$ is called a Lorentzian TransSasakian manifold if it admits a $(1,1)$ tensor field $\phi$, a contravariant vector field $\xi$, a covariant vector field $\eta$ and the Lorentzian metric $\mathrm{g}$ which satisfy

$$
\begin{gathered}
\eta(\xi)=-1, \\
\phi^{2}=I+\eta \otimes \xi, \\
g(\phi X, \phi Y)=g(X, Y)+\eta(X) \eta(Y), \\
g(X, \xi)=\eta(X), \phi \xi=0, \eta(\phi X)=0, \\
\left(\nabla_{X} \phi\right) Y=\alpha(g(X, Y) \xi-\eta(Y) X)+\beta(g(\phi X, Y) \xi-\eta(Y) \phi X),
\end{gathered}
$$

for all $X, Y \in T(M)$.

Also a Lorentzian Trans-Sasakian manifold $M$ satisfies

$$
\begin{gathered}
\nabla_{X} \xi=-\alpha(\phi X)-\beta(X+\eta(X) \xi), \\
\left(\nabla_{X} \eta\right) Y=\alpha g(\phi X, Y)+\beta g(\phi X, \phi Y),
\end{gathered}
$$

where $\nabla$ denotes the operator of covariant differentation with respect to the Lorentzian metric $g$.

If $\alpha=0$ and $\beta \varepsilon \mathbb{R}$, the set of real numbers, then the manifold reduces to a Lorentzian $\beta$-Kenmotsu manifold studied by Funda Yaliniz, Yildiz, and Turan [20]. If $\beta=0$ and $\alpha \varepsilon \mathbb{R}$, then the manifold reduces to a Lorentzian $\alpha$-Sasakian manifold studied by Yildiz, Turan and Murathan [21]. If $\alpha=0$ and $\beta=1$, then the manifold reduces to a Lorentzian Kenmotsu manifold introduced by Mihai, Oiaga and Rosca [15]. Furthermore, if $\beta=0$ and $\alpha=1$, then the manifold reduces to a Lorentzian Sasakian manifold studied by Ikawa and Erdogan [15]. Also Lorentzian para contact manifolds were introduced by Matsumoto [12] and further studied by the authors ([13],[14],[16]). Trans Lorentzian para Sasakian manifolds have been used by Gill and Dube [7]. 


\section{SOME BASIC RESULTS}

In this section, we prove some Lemmas which are needed in the rest of the sections.

Lemma 3.1. In a Lorentzian Trans-Sasakian manifold, we have

$$
\begin{aligned}
R(X, Y) \xi= & \left(\alpha^{2}+\beta^{2}\right)(\eta(Y) X-\eta(X) Y) \\
& +2 \alpha \beta(\eta(Y) \phi X-\eta(X) \phi Y)+(Y \alpha) \phi X \\
& -(X \alpha) \phi Y+(Y \beta) \phi^{2} X-(X \beta) \phi^{2} Y,
\end{aligned}
$$

where $R$ is the curvature tensor.

Proof. We have

$$
\begin{aligned}
\nabla_{X} \nabla_{Y} \xi= & \nabla_{X}(-\alpha(\phi Y)-\beta(Y+\eta(Y) \xi)) \\
= & -(X \alpha) \phi Y-\alpha \nabla_{X}(\phi Y)-(X \beta) \phi^{2} Y \\
& -\beta \nabla_{X} Y-\beta(X \eta(Y)) \xi+\alpha \beta \eta(Y) \phi X \\
& +\beta^{2} \eta(Y) X+\beta^{2} \eta(X) \eta(Y) \xi,
\end{aligned}
$$

where (2.2) and (2.6) have been used. Hence, in view of the above equation and (2.6), we get

$$
\begin{aligned}
R(X, Y) \xi= & \nabla_{X} \nabla_{Y} \xi-\nabla_{Y} \nabla_{X} \xi-\nabla_{[X, Y]} \xi \\
= & -(X \alpha) \phi Y+(Y \alpha) \phi X-\alpha\left(\left(\nabla_{X} \phi Y\right)-\left(\nabla_{Y} \phi X\right)\right) \\
& -(X \beta) \phi^{2} Y+(Y \beta) \phi^{2} X-\beta\left(\left(\nabla_{X} \eta\right) Y-\left(\nabla_{Y} \eta\right) X\right) \xi \\
& +\alpha \beta(\eta(Y) \phi X-\eta(X) \phi Y)+\beta^{2}(\eta(Y) X-\eta(X) Y),
\end{aligned}
$$

which in view of (2.5) and (2.7) gives (3.1).

Lemma 3.2. For a Lorentzian Trans-Sasakian manifold, we have

$$
\eta(R(X, Y) Z)=\left(\alpha^{2}+\beta^{2}\right)(g(X, Z) \eta(Y)-g(Y, Z) \eta(X)) .
$$

Proof. We have from (3.1),

$$
\begin{aligned}
g(R(X, Y) \xi, Z)= & \left(\alpha^{2}+\beta^{2}\right)(\eta(Y) g(X, Z)-\eta(X) g(Y, Z)) \\
& +2 \alpha \beta(\eta(Y) g(\phi X, Z)-\eta(X) g(\phi Y, Z))+(Y \alpha) g(\phi X, Z) \\
& -(X \alpha) g(\phi Y, Z)+(Y \beta) g\left(\phi^{2} X, Z\right)-(X \beta) g\left(\phi^{2} Y, Z\right),
\end{aligned}
$$

Now interchanging $\xi$ and $Z$ in the above equation, we get

$$
\begin{aligned}
-g(R(X, Y) Z, \xi)= & \left(\alpha^{2}+\beta^{2}\right)(g(Y, Z) \eta(X)-g(X, Z) \eta(Y)) \\
& +2 \alpha \beta(\eta(Y) g(\phi X, \xi)-\eta(X) g(\phi Y, \xi))+(Y \alpha) g(\phi X, \xi) \\
& -(X \alpha) g(\phi Y, \xi)+(Y \beta) g\left(\phi^{2} X, \xi\right)-(X \beta) g\left(\phi^{2} Y, \xi\right) .
\end{aligned}
$$

After simplification, we find,

$$
g(R(X, Y) Z, \xi)=\left(\alpha^{2}+\beta^{2}\right)(g(X, Z) \eta(Y)-g(Y, Z) \eta(X)),
$$


which gives (3.2).

Lemma 3.3. For a Lorentzian Trans-Sasakian manifold, we have

$$
R(\xi, Y) \xi=\left(\alpha^{2}+\beta^{2}-\xi \beta\right) \phi^{2} Y+(2 \alpha \beta-\xi \alpha) \phi Y .
$$

Proof. Replacing $X$ by $\xi$ in (3.1), we get (3.3).

Lemma 3.4. In a $(2 n+1)$ - dimensional Lorentzian Trans-Sasakian manifold, we have

$$
\begin{aligned}
S(X, \xi)= & \left(2 n\left(\alpha^{2}+\beta^{2}\right)-\xi \beta\right) \eta(X)+(2 n-1)(X \beta) \\
& -(\phi X) \alpha+\psi(2 \alpha \beta \eta(X)+X \alpha), \\
Q \xi= & \left(2 n\left(\alpha^{2}+\beta^{2}\right)-\xi \beta\right) \xi+(2 n-1) \operatorname{grad} \beta \\
& -\phi(\operatorname{grad} \alpha)+\psi(2 \alpha \beta \xi+\operatorname{grad} \alpha),
\end{aligned}
$$

where $S$ is the Ricci curvature and $Q$ is the Ricci operator given by

$$
S(X, Y)=g(Q X, Y) \text { and } \psi=\sum_{i=1}^{2 n+1} \epsilon_{i} g\left(\phi e_{i}, e_{i}\right) .
$$

Proof. Let $M$ be an $(2 n+1)$ - dimensional Lorentzian Trans-Sasakian manifold. Then the Ricci tensor $S$ of the manifold M is defined by

$$
S(X, Y)=\sum_{i=1}^{2 n+1} \epsilon_{i} g\left(R\left(e_{i}, X\right) Y, e_{i}\right)
$$

where $\epsilon_{i}=g\left(e_{i}, e_{i}\right), \epsilon_{i}= \pm 1$. From (3.1), we have

$$
\begin{aligned}
S(X, \xi)= & \left(\alpha^{2}+\beta^{2}\right)\left[\eta(X) \sum_{i=1}^{2 n+1} g\left(e_{i}, e_{i}\right) g\left(e_{i}, e_{i}\right)-\sum_{i=1}^{2 n+1} \eta\left(e_{i}\right) g\left(e_{i}, e_{i}\right) g\left(X, e_{i}\right)\right] \\
& +2 \alpha \beta\left[\eta(X) \sum_{i=1}^{2 n+1} g\left(e_{i}, e_{i}\right) g\left(\phi e_{i}, e_{i}\right)-\sum_{i=1}^{2 n+1} \eta\left(e_{i}\right) g\left(e_{i}, e_{i}\right) g\left(\phi X, e_{i}\right)\right] \\
& -\sum_{i=1}^{2 n+1}\left(e_{i} \alpha\right) g\left(e_{i}, e_{i}\right) g\left(\phi X, e_{i}\right)+\sum_{i=1}^{2 n+1}(X \alpha) g\left(e_{i}, e_{i}\right) g\left(\phi e_{i}, e_{i}\right) \\
& -\sum_{i=1}^{2 n+1}\left(e_{i} \beta\right) g\left(e_{i}, e_{i}\right) g\left(\phi^{2} X, e_{i}\right)+\sum_{i=1}^{2 n+1}(X \beta) g\left(e_{i}, e_{i}\right) g\left(\phi^{2} e_{i}, e_{i}\right) \\
= & \left(2 n\left(\alpha^{2}+\beta^{2}\right)-\xi \beta\right) \eta(X)+(2 n-1)(X \beta) \\
& -(\phi X) \alpha+\psi(2 \alpha \beta \eta(X)+X \alpha)
\end{aligned}
$$

and hence from (3.4), we get (3.5). 
Remark 3.5. If in a $(2 n+1)$ - dimensional Lorentzian Trans-Sasakian manifold of type $(\alpha, \beta)$ we consider $\phi(\operatorname{grad} \alpha)=(2 n-1) \operatorname{grad} \beta$, then

$$
\begin{aligned}
\xi \beta & =g(\xi, \operatorname{grad} \beta)=\frac{1}{2 n-1} g(\xi, \phi(\operatorname{grad} \alpha)) \\
& =\frac{1}{2 n-1} \eta(\phi(\operatorname{grad} \alpha))=0
\end{aligned}
$$

and

$$
\begin{aligned}
X \beta & =g(X, \operatorname{grad} \beta)=\frac{1}{2 n-1} g(X, \phi(\operatorname{grad} \alpha)) \\
& =\frac{1}{2 n-1} g(\phi X,(\operatorname{grad} \alpha))=\frac{1}{2 n-1}(\phi X) \alpha
\end{aligned}
$$

and hence (3.4) and (3.5) are reduced to

$$
S(X, \xi)=2 n\left(\alpha^{2}+\beta^{2}\right) \eta(X)+\psi(2 \alpha \beta \eta(X)+X \alpha)
$$

and

$$
Q \xi=\left(2 n\left(\alpha^{2}+\beta^{2}\right)-\xi \beta\right) \xi+\psi(2 \alpha \beta \xi+\operatorname{grad} \alpha)
$$

respectively.

\section{Conformally Flat Lorentzian Trans-Sasakian manifolds}

In this section we consider conformally flat Lorentzian Trans-Sasakian manifold $M^{2 n+1}(\phi, \xi, \eta, g)(n>1)$. The conformal curvature tensor $C$ is given by

$$
\begin{aligned}
C(X, Y) Z= & R(X, Y) Z-\frac{1}{2 n-1}[S(Y, Z) X-S(X, Z) Y+g(Y, Z) Q X \\
& -g(X, Z) Q Y]+\frac{r}{(2 n)(2 n-1)}[g(Y, Z) X-g(X, Z) Y]
\end{aligned}
$$

where $r$ is the scalar curvature of $M$.

For conformally flat manifold, we have $C(X, Y) Z=0$ for $n>1$ and hence from (4.1) we have

$$
\begin{aligned}
\widetilde{R}(X, Y, Z, W)= & \frac{1}{2 n-1}[S(Y, Z) g(X, W)-S(X, Z) g(Y, W) \\
& +g(Y, Z) S(X, W)-g(X, Z) S(Y, W)] \\
& -\frac{r}{(2 n)(2 n-1)}[g(Y, Z) g(X, W)-g(X, Z) g(Y, W)],
\end{aligned}
$$

where $g(R(X, Y) Z, U)=\widetilde{R}(X, Y, Z, U)$. Setting $W=\xi$ in (4.2) we get 


$$
\begin{aligned}
\eta(R(X, Y) Z)= & \frac{1}{2 n-1}[S(Y, Z) \eta(X)-S(X, Z) \eta(Y) \\
& +g(Y, Z) S(X, \xi)-g(X, Z) S(Y, \xi)] \\
& -\frac{r}{(2 n)(2 n-1)}[g(Y, Z) \eta(X)-g(X, Z) \eta(Y)] .
\end{aligned}
$$

Replacing $Y$ by $\xi$ in (4.3) and using (3.2) and (3.6), we get

$$
\begin{aligned}
S(X, Z)= & {\left[\left(\alpha^{2}+\beta^{2}\right)(1-4 n)+\frac{r}{2 n}+(\xi \alpha-2 \alpha \beta) \psi\right] g(X, Z) } \\
& +\left[\left(\alpha^{2}+\beta^{2}\right)(1-6 n)+\frac{r}{2 n}-4 \alpha \beta \psi\right] \eta(X) \eta(Z) \\
& -[\eta(Z)(X \alpha)+\eta(X)(Z \alpha)] \psi .
\end{aligned}
$$

This leads to the following:

Theorem 4.1. A conformally flat Lorentzian Trans Sasakian manifold $M^{2 n+1}$ $(\phi, \xi, \eta, g)(n>1)$ is an $\eta-$ Einstein manifold provided $\psi=$ trace $\phi=o$ and $\phi(\operatorname{grad} \alpha)=(2 n-1) \operatorname{grad} \beta$.

Corollary 1. A conformally flat Lorentzian $\beta$ - Kenmotsu manifold $M^{2 n+1}(\phi, \xi, \eta, g)$ $(n>1)$ is an $\eta-$ Einstein manifold.

\section{Three- Dimensional Lorentzian Trans- Sasakian manifolds}

Since the conformal curvature tensor vanishes in a three-dimensional Riemannian manifold, therefore we get

$$
\begin{aligned}
R(X, Y) Z= & g(Y, Z) Q X-g(X, Z) Q Y+S(Y, Z) X-S(X, Z) Y \\
& -\frac{r}{2}[g(Y, Z) X-g(X, Z) Y],
\end{aligned}
$$

where $Q$ is the Ricci operator, that is, $g(Q X, Y)=S(X, Y)$ and $r$ is the scalar curvature of the manifold.

From Lemma 2.4, in a three- dimensional Lorentzian Trans-Sasakian manifold we have

$$
\begin{aligned}
S(X, \xi)= & \left(2\left(\alpha^{2}+\beta^{2}\right)-\xi \beta\right) \eta(X)+(X \beta) \\
& -(\phi X) \alpha+\psi(2 \alpha \beta \eta(X)+X \alpha), \\
Q \xi= & \left(2\left(\alpha^{2}+\beta^{2}\right)-\xi \beta\right) \xi+\operatorname{grad} \beta \\
& -\phi(\operatorname{grad} \alpha)+\psi(2 \alpha \beta \xi+\operatorname{grad} \alpha) .
\end{aligned}
$$


Now, in the following theorem, we obtain an expression for Ricci operator in a three-dimensional Lorentzian Trans-Sasakian manifold.

Theorem 5.1. In a three- dimensional Lorentzian Trans Sasakian manifold, the Ricci operator is given by

$$
\begin{aligned}
Q X= & \left(\frac{r}{2}+\xi \beta-\left(\alpha^{2}+\beta^{2}\right)+\psi(\xi \alpha-2 \alpha \beta)\right) X \\
& +\left(\frac{r}{2}+\xi \beta-3\left(\alpha^{2}+\beta^{2}\right)-4 \alpha \beta \psi\right) \eta(X) \xi \\
& -\eta(X)(\operatorname{grad} \beta-\phi(\operatorname{grad} \alpha)+\psi(\operatorname{grad} \alpha))-(X \beta-(\phi X) \alpha+\psi(X \alpha)) \xi \\
& +(2 \alpha \beta-\xi \alpha) \phi X
\end{aligned}
$$

Proof. For a three- dimensional Lorentzian Trans Sasakian manifold, from (5.1) and (5.2), we have

$$
\begin{aligned}
R(X, Y) \xi= & \eta(Y) Q X-\eta(X) Q Y \\
& -\left(\frac{r}{2}+\xi \beta-2\left(\alpha^{2}+\beta^{2}\right)-2 \alpha \beta \psi\right)[X \eta(Y)-Y \eta(X)] \\
& +(Y \beta-(\phi Y) \alpha+(Y \alpha) \psi) X-(X \beta-(\phi X) \alpha+(X \alpha) \psi) Y .(5.5)
\end{aligned}
$$

In view of (3.1) and (5.5), we obtain

$$
\begin{aligned}
2 \alpha \beta(\eta(Y) \phi X-\eta(X) \phi Y)+ & (Y \alpha) \phi X-(X \alpha) \phi Y+(Y \beta) \phi^{2} X-(X \beta) \phi^{2} Y \\
= & \eta(Y) Q X-\eta(X) Q Y-\left(\frac{r}{2}+\xi \beta-\left(\alpha^{2}+\beta^{2}\right)-2 \alpha \beta \psi\right) \\
& {[\eta(Y) X-\eta(X) Y]+(Y \beta-(\phi Y) \alpha+(Y \alpha) \psi) X } \\
& -(X \beta-(\phi X) \alpha+(X \alpha) \psi) Y .
\end{aligned}
$$

Putting $Y=\xi$ in the above equation, we get (5.4) .

Corollary 2. In a three- dimensional Lorentzian Trans Sasakian manifold, Ricci tensor and curvature tensor are given respectively by

$$
\begin{aligned}
S(X, Y)= & \left(\frac{r}{2}+\xi \beta-\left(\alpha^{2}+\beta^{2}\right)+\psi(\xi \alpha-2 \alpha \beta)\right) g(X, Y) \\
& +\left(\frac{r}{2}+\xi \beta-3\left(\alpha^{2}+\beta^{2}\right)-4 \alpha \beta \psi\right) \eta(X) \eta(Y) \\
& +\eta(X)[-Y \beta+(\phi Y) \alpha-\psi(Y \alpha)]-\eta(Y)(X \beta-(\phi X) \alpha+\psi(X \alpha)) \\
& +(2 \alpha \beta-\xi \alpha) g(\phi X, Y)
\end{aligned}
$$


and

$$
\begin{aligned}
R(X, Y) Z= & \left(\frac{r}{2}+2 \xi \beta-2\left(\alpha^{2}+\beta^{2}\right)+2 \psi(\xi \alpha-2 \alpha \beta)\right)[g(Y, Z) X-g(X, Z) Y] \\
& +g(Y, Z)\left[\left(\frac{r}{2}+\xi \beta-3\left(\alpha^{2}+\beta^{2}\right)-4 \alpha \beta \psi\right) \eta(X) \xi\right. \\
& +\eta(X)(\phi(\operatorname{grad} \alpha)-\psi(\operatorname{grad} \alpha)-\operatorname{grad} \beta)-(X \beta-(\phi X) \alpha+\psi(X \alpha)) \xi] \\
& +g(X, Z)\left[\left(\frac{r}{2}+\xi \beta-3\left(\alpha^{2}+\beta^{2}\right)-4 \alpha \beta \psi\right) \eta(Y) \xi\right. \\
& +\eta(Y)(\phi(g r a d \alpha)-\psi(\operatorname{grad} \alpha)-\operatorname{grad} \beta)-(Y \beta-(\phi Y) \alpha+\psi(Y \alpha)) \xi] \\
& +\left[\left(\frac{r}{2}+\xi \beta-3\left(\alpha^{2}+\beta^{2}\right)-4 \alpha \beta \psi\right) \eta(Y) \eta(Z)\right. \\
& +\eta(Y)(-Z \beta+(\phi Z) \alpha-\psi(Z \alpha))-\eta(Z)(Y \beta-(\phi Y) \alpha+\psi(Y \alpha))] X \\
& -\left[\left(\frac{r}{2}+\xi \beta-3\left(\alpha^{2}+\beta^{2}\right)-4 \alpha \beta \psi\right) \eta(X) \eta(Z)\right. \\
& +\eta(X)(-Z \beta+(\phi Z) \alpha-\psi(Z \alpha))-\eta(Z)(X \beta-(\phi X) \alpha+\psi(X \alpha))] Y \\
& +(2 \alpha \beta-\xi \alpha)[g(\phi Y, Z) X-g(\phi X, Z) Y] .
\end{aligned}
$$

Equation (5.6) follows from (5.4). Using (5.4) and (5.6) in (5.1), the curvature tensor in a three-dimensional Lorentzian Trans-Sasakian manifold is given by (5.7).

\section{LoCALly $\phi$ - SYMmetriC THREE-Dimensional LORENTZIAN}

TRANS-SASAKIAN MANIFOLDS WITH trace $\phi=\psi=0$

The notion of locally $\phi$-symmetry was first introduced by T.Takahashi [19] on a Sasakian manifold. In this paper we study locally $\phi$-symmetric three-dimensional Lorentzian Trans-Sasakian manifolds.

Definition 6.1. A three-dimensional Lorentzian Trans-Sasakian manifold is said to be locally $\phi$ - symmetric if

$$
\phi^{2}\left(\nabla_{W} R\right)(X, Y) Z=0
$$

where W,X,Y,Z are horizontal vector fields, that is $\mathrm{W}, \mathrm{X}, \mathrm{Y}, \mathrm{Z}$ are orthogonal to $\xi$. 
Let $\mathrm{M}$ be a three- dimensional Lorentzian Trans-Sasakian manifold with trace $\phi$ $=\psi=0$. Then its curvature tensor is given by

$$
\begin{aligned}
R(X, Y) Z= & \left(\frac{r}{2}+2 \xi \beta-2\left(\alpha^{2}+\beta^{2}\right)\right)[g(Y, Z) X-g(X, Z) Y] \\
& +g(Y, Z)\left[\left(\frac{r}{2}+\xi \beta-3\left(\alpha^{2}+\beta^{2}\right)\right) \eta(X) \xi\right. \\
& +\eta(X)(\phi(\operatorname{grad} \alpha)-\operatorname{grad} \beta)-(X \beta-(\phi X) \alpha) \xi] \\
& +g(X, Z)\left[\left(\frac{r}{2}+\xi \beta-3\left(\alpha^{2}+\beta^{2}\right)\right) \eta(Y) \xi\right. \\
& +\eta(Y)(\phi(\operatorname{grad} \alpha)-\operatorname{grad} \beta)-(Y \beta-(\phi Y) \alpha) \xi] \\
& +\left[\left(\frac{r}{2}+\xi \beta-3\left(\alpha^{2}+\beta^{2}\right)\right) \eta(Y) \eta(Z)\right. \\
& +\eta(Y)(-Z \beta+(\phi Z \alpha))-\eta(Z)(Y \beta-(\phi Y) \alpha)] X \\
& -\left[\left(\frac{r}{2}+\xi \beta-3\left(\alpha^{2}+\beta^{2}\right)\right) \eta(X) \eta(Z)\right. \\
& +\eta(X)(-Z \beta+(\phi Z \alpha))-\eta(Z)(X \beta-(\phi X) \alpha)] Y \\
& +(2 \alpha \beta-\xi \alpha)[g(\phi Y, Z) X-g(\phi X, Z) Y] .
\end{aligned}
$$

Differentiating (6.2) we get

$$
\begin{aligned}
\left(\nabla_{W} R\right)(X, Y) Z= & {\left[\frac{d r(W)}{2}+2\left(\nabla_{W}(\xi \beta)\right)-4(d \alpha(W)+d \beta(W))\right] } \\
& {[g(Y, Z) X-g(X, Z) Y]+g(Y, Z)\left[\left(\frac{d r(W)}{2}+\left(\nabla_{W}(\xi \beta)\right)\right.\right.} \\
& -6(d \alpha(W)+d \beta(W)) \eta(X) \xi+\left(\frac{r}{2}+\xi \beta-3\left(\alpha^{2}\right.\right. \\
& \left.\left.+\beta^{2}\right)\right)\left(\left(\nabla_{W} \eta\right)(X) \xi+\eta(X)\left(\nabla_{W} \xi\right)\right) \\
& +\left(\nabla_{W} \eta\right)(X)(\phi(g r a d \alpha)-g r a d \beta)+\eta(X)\left(\nabla_{W}(\phi(g r a d \alpha)-g r a d \beta)\right) \\
& \left.+\left(\nabla_{W}(X \beta-(\phi X) \alpha)\right) \xi+(X \beta-(\phi X) \alpha) \nabla_{W} \xi\right] \\
& -g(X, Z)\left[\left(\frac{d r(W)}{2}+\left(\nabla_{W}(\xi \beta)\right)-6(d \alpha(W)+d \beta(W))\right) \eta(Y) \xi\right. \\
& +\left(\frac{r}{2}+\xi \beta-3\left(\alpha^{2}+\beta^{2}\right)\right)\left(\left(\nabla_{W} \eta\right)(Y) \xi+\eta(Y)\left(\nabla_{W} \xi\right)\right) \\
& +\left(\nabla_{W} \eta\right)(Y)(\phi(g r a d \alpha)-g r a d \beta)+\eta(Y)\left(\nabla_{W}(\phi(g r a d \alpha)-g r a d \beta)\right) \\
& \left.+\left(\nabla_{W}(Y \beta-(\phi Y) \alpha)\right) \xi+(Y \beta-(\phi Y) \alpha) \nabla_{W} \xi\right] \\
& -Y\left[\left(\nabla_{W}(Y \beta-(\phi Y) \alpha)\right) \eta(Z)+(Y \beta-(\phi Y) \alpha)\left(\nabla_{W} \eta\right) Z\right. \\
& \left.+\nabla_{W}(Z \beta-(\phi Z) \alpha)\right) \eta(Y)+(Z \beta-(\phi Z) \alpha)\left(\nabla_{W} \eta\right) Y \\
& -\left(\frac{d r(W)}{2}+\left(\nabla_{W}(\xi \beta)\right)-6(d \alpha(W)+d \beta(W))\right) \eta(Y) \eta(Z)
\end{aligned}
$$




$$
\begin{aligned}
& -\left(\frac{r}{2}+\xi \beta-3\left(\alpha^{2}+\beta^{2}\right)\right) \\
& \left.\left(\left(\nabla_{W} \eta\right) Y \eta(Z)+\eta(Y)\left(\nabla_{W} \eta\right) Z\right)\right] \\
& +X\left[\left(\nabla_{W}(X \beta-(\phi X) \alpha)\right) \eta(Z)+(X \beta-(\phi X) \alpha)\left(\nabla_{W} \eta\right) Z\right. \\
& \left.+\nabla_{W}(Z \beta-(\phi Z) \alpha)\right) \eta(X)+(Z \beta-(\phi Z) \alpha)\left(\nabla_{W} \eta\right) X \\
& -\left(\frac{d r(W)}{2}+\left(\nabla_{W}(\xi \beta)\right)-6(d \alpha(W)+d \beta(W))\right) \eta(X) \eta(Z) \\
& -\left(\frac{r}{2}+\xi \beta-3\left(\alpha^{2}+\beta^{2}\right)\right) \\
& \left.\left(\left(\nabla_{W} \eta\right) X \eta(Z)+\eta(X)\left(\nabla_{W} \eta\right) Z\right)\right] \\
& +\left(2\left(\nabla_{W}(\alpha \beta)\right)-\left(\nabla_{W}(\xi \alpha)\right)\right)[g(\phi Y, Z) X-g(\phi X, Z) Y] .
\end{aligned}
$$

Suppose that $\alpha$ and $\beta$ are constants and $\mathrm{X}, \mathrm{Y}, \mathrm{Z}, \mathrm{W}$ are orthogonal to $\xi$. Then using $\phi \xi=0$ and (6.1), we get

$$
\phi^{2}\left(\nabla_{W} R\right)(X, Y) Z=\left(\frac{d r(W)}{2}\right)[g(Y, Z) X-g(X, Z) Y] .
$$

Thus we can state the following:

Theorem 6.2. A three-dimensional Lorentzian Trans-Sasakian manifold of type $(\alpha, \beta)$ is locally $\phi$ - symmetric if and only if the scalar curvature $r$ is constant provided $\alpha$ and $\beta$ are constants.

\section{EXAMPLES}

Example 7.1: We consider the three-dimensional manifold $M=\left\{(x, y, z) \varepsilon \mathbb{R}^{3}, z \neq\right.$ $0\}$, where $(x, y, z)$ are standard co-ordinate of $\mathbb{R}^{3}$.

The vector fields

$$
e_{1}=z\left(\frac{\partial}{\partial x}+y \frac{\partial}{\partial z}\right), \quad e_{2}=z \frac{\partial}{\partial y}, \quad e_{3}=\frac{\partial}{\partial z}
$$

are linearly independent at each point of $M$.

Let $g$ be the Riemannian metric defined by

$$
\begin{gathered}
g\left(e_{1}, e_{3}\right)=g\left(e_{1}, e_{2}\right)=g\left(e_{2}, e_{3}\right)=0, \\
g\left(e_{1}, e_{1}\right)=g\left(e_{2}, e_{2}\right)=1, g\left(e_{3}, e_{3}\right)=-1 .
\end{gathered}
$$

Let $\eta$ be the 1-form defined by $\eta(Z)=g\left(Z, e_{3}\right)$ for any $Z \varepsilon \chi(M)$.

Let $\phi$ be the $(1,1)$ tensor field defined by

$$
\phi\left(e_{1}\right)=-e_{2}, \quad \phi\left(e_{2}\right)=-e_{1}, \quad \phi\left(e_{3}\right)=0 .
$$

Then using the linearity of $\phi$ and $g$, we have

$$
\begin{gathered}
\eta\left(e_{3}\right)=-1, \\
\phi^{2} Z=Z+\eta(Z) e_{3},
\end{gathered}
$$


for any $Z, W \varepsilon \chi(M)$.

$$
g(\phi Z, \phi W)=g(Z, W)+\eta(Z) \eta(W)
$$

Then for $e_{3}=\xi$, the structure $(\phi, \xi, \eta, g)$ defines an Lorentzian structure on $M$.

Let $\nabla$ be the Levi-Civita connection with respect to metric $g$ and $R$ be the curvature tensor of $g$. Then we have

$$
\left[e_{1}, e_{2}\right]=y e_{2}-z^{2} e_{3} \quad,\left[e_{1}, e_{3}\right]=-\frac{1}{z} e_{1} \quad \text { and } \quad\left[e_{2}, e_{3}\right]=-\frac{1}{z} e_{2} .
$$

Taking $e_{3}=\xi$ and using Koszul formula for the Riemannian metric $g$, we can easily calculate

$$
\begin{gathered}
\nabla_{e_{1}} e_{3}=-\frac{1}{z} e_{1}+\frac{1}{z^{2}} e_{2}, \quad \nabla_{e_{1}} e_{2}=-\frac{1}{2} z^{2} e_{3} \\
\nabla_{e_{1}} e_{1}=-\frac{1}{z} e_{3}, \nabla_{e_{2}} e_{3}=-\frac{1}{z} e_{2}+\frac{1}{2} z^{2} e_{1} \\
\nabla_{e_{2}} e_{2}=y e_{1}-\frac{1}{z} e_{3}, \quad \nabla_{e_{2}} e_{1}=\frac{1}{2} z^{2} e_{3}-y e_{2}, \\
\nabla_{e_{3}} e_{3}=0, \quad \nabla_{e_{3}} e_{2}=\frac{1}{2} z^{2} e_{1}, \quad \nabla_{e_{3}} e_{1}=-\frac{1}{2} z^{2} e_{2} .
\end{gathered}
$$

From the above it can be easily seen that $(\phi, \xi, \eta, g)$ is an Lorentzian Trans-Sasakian structure on $M$. Consequently $M^{3}(\phi, \xi, \eta, g)$ is an Lorentzian Trans-Sasakian manifold with $\alpha=\frac{1}{2} z^{2} \neq 0$ and $\beta=\frac{1}{z} \neq 0$.

Example 7.2: We consider the three-dimensional manifold $M=\{(x, y, z) \in$ $\left.\mathbb{R}^{3}, z \neq 0\right\}$, where $(x, y, z)$ are standard co-ordinate of $\mathbb{R}^{3}$.

The vector fields

$$
e_{1}=z \frac{\partial}{\partial x}, \quad e_{2}=z \frac{\partial}{\partial y}, \quad e_{3}=z \frac{\partial}{\partial z}
$$

are linearly independent at each point of $M$.

Let $g$ be the Riemannian metric defined by

$$
\begin{gathered}
g\left(e_{1}, e_{3}\right)=g\left(e_{1}, e_{2}\right)=g\left(e_{2}, e_{3}\right)=0, \\
g\left(e_{1}, e_{1}\right)=g\left(e_{2}, e_{2}\right)=1, g\left(e_{3}, e_{3}\right)=-1
\end{gathered}
$$

that is, the form of the metric becomes

$$
g=\frac{d x^{2}+d y^{2}-d z^{2}}{z^{2}}
$$

Let $\eta$ be the 1-form defined by $\eta(Z)=g\left(Z, e_{3}\right)$ for any $Z \varepsilon \chi(M)$.

Let $\phi$ be the $(1,1)$ tensor field defined by

$$
\phi\left(e_{1}\right)=-e_{2}, \quad \phi\left(e_{2}\right)=-e_{1}, \quad \phi\left(e_{3}\right)=0 .
$$

Then using the linearity of $\phi$ and $g$, we have

$$
\begin{gathered}
\eta\left(e_{3}\right)=-1, \\
\phi^{2} Z=Z+\eta(Z) e_{3},
\end{gathered}
$$


for any $Z, W \in \chi(M)$.

$$
g(\phi Z, \phi W)=g(Z, W)+\eta(Z) \eta(W),
$$

Then for $e_{3}=\xi$, the structure $(\phi, \xi, \eta, g)$ defines an Lorentzian structure on $M$.

Let $\nabla$ be the Levi-Civita connection with respect to metric $g$. Then we have

$$
\begin{aligned}
{\left[e_{1}, e_{3}\right] } & =e_{1} e_{3}-e_{3} e_{1} \\
& =z \frac{\partial}{\partial x}\left(z \frac{\partial}{\partial z}\right)-z \frac{\partial}{\partial z}\left(z \frac{\partial}{\partial x}\right) \\
& =z^{2} \frac{\partial^{2}}{\partial x \partial z}-z^{2} \frac{\partial^{2}}{\partial z \partial x}-z \frac{\partial}{\partial x} \\
& =-e_{1} .
\end{aligned}
$$

Similarly

$$
\left[e_{1}, e_{2}\right]=0 \quad \text { and } \quad\left[e_{2}, e_{3}\right]=-e_{2}
$$

The Riemannian connection $\nabla$ of the metric $g$ is given by

$$
\begin{aligned}
2 g\left(\nabla_{X} Y, Z\right) & =X g(Y, Z)+Y g(Z, X)-Z g(X, Y) \\
& -g(X,[Y, Z])-g(Y,[X, Z])+g(Z,[X, Y]),
\end{aligned}
$$

which known as Koszul's formula.

Using (7.1) we have

$$
\begin{aligned}
2 g\left(\nabla_{e_{1}} e_{3}, e_{1}\right) & =-2 g\left(e_{1}, e_{1}\right) \\
& =2 g\left(-e_{1}, e_{1}\right) .
\end{aligned}
$$

Again by (7.1)

$$
2 g\left(\nabla_{e_{1}} e_{3}, e_{2}\right)=0=2 g\left(-e_{1}, e_{2}\right)
$$

and

$$
2 g\left(\nabla_{e_{1}} e_{3}, e_{3}\right)=0=2 g\left(-e_{1}, e_{3}\right) .
$$

From (7.2), (7.3) and (7.4) we obtain

$$
2 g\left(\nabla_{e_{1}} e_{3}, X\right)=2 g\left(-e_{1}, X\right)
$$

for all $X \varepsilon \chi(M)$.

Thus

$$
\nabla_{e_{1}} e_{3}=-e_{1} .
$$

Therefore, (7.1) further yields

$$
\begin{array}{cl}
\nabla_{e_{1}} e_{3}=-e_{1}, & \nabla_{e_{1}} e_{2}=0, \quad \nabla_{e_{1}} e_{1}=-e_{3}, \\
\nabla_{e_{2}} e_{3}=-e_{2}, & \nabla_{e_{2}} e_{2}=-e_{3}, \quad \nabla_{e_{2}} e_{1}=0 \\
\nabla_{e_{3}} e_{3}=0, & \nabla_{e_{3}} e_{2}=0, \quad \nabla_{e_{3}} e_{1}=0 .
\end{array}
$$

(7.5) tells us that the manifold satisfies (1.3) for $\alpha=0, \beta=1$ and $\xi=e_{3}$. Hence the manifold is a Lorentzian Trans-Sasakian manifold of type $(0,1)$. It is known that

$$
R(X, Y) Z=\nabla_{X} \nabla_{Y} Z-\nabla_{Y} \nabla_{X} Z-\nabla_{[X, Y]} Z
$$


With the help of the above results and using (7.6) it can be easily verified that

$$
\begin{gathered}
R\left(e_{1}, e_{2}\right) e_{3}=0, \quad R\left(e_{2}, e_{3}\right) e_{3}=-e_{2}, \quad R\left(e_{1}, e_{3}\right) e_{3}=-e_{1}, \\
R\left(e_{1}, e_{2}\right) e_{2}=-e_{1}, \quad R\left(e_{2}, e_{3}\right) e_{2}=-e_{3}, \quad R\left(e_{1}, e_{3}\right) e_{2}=0, \\
R\left(e_{1}, e_{2}\right) e_{1}=e_{2}, \quad R\left(e_{2}, e_{3}\right) e_{1}=0, \quad R\left(e_{1}, e_{3}\right) e_{1}=-e_{3} .
\end{gathered}
$$

From the expression of the curvature tensor it follows that the manifold is of constant curvature -1 . Hence the manifold is locally $\phi$-symmetric. Also from the above expressions of the curvature tensor, we obtain

$$
\begin{aligned}
S\left(e_{1}, e_{1}\right) & =g\left(R\left(e_{1}, e_{2}\right) e_{2}, e_{1}\right)+g\left(R\left(e_{1}, e_{3}\right) e_{3}, e_{1}\right) \\
& =-2 .
\end{aligned}
$$

Similarly, we have

$$
S\left(e_{2}, e_{2}\right)=-2, S\left(e_{3}, e_{3}\right)=2 .
$$

Therefore,

$$
r=S\left(e_{1}, e_{1}\right)+S\left(e_{2}, e_{2}\right)-S\left(e_{3}, e_{3}\right)=-6 .
$$

Thus the scalar curvature $r$ is constant. Hence Theorem 6.1 is verified.

\section{REFERENCES}

[1] Blair, D. E., Contact manifolds in Riemannian geometry, Lecture Notes in Math., Springer Verlag, (509)1976.

[2] Blair, D. E.and Oubina, J.A., Conformal and related changes of metric on the product of two almost contact metric manifolds, Publ. Mat.34(1)(1990), 199 - 207.

[3] Chinea,D. and Gonzales, C., Curvature relations in trans-sasakian manifolds, (Spanish), in "Proceedings of the XIIth Portuguese-Spanish Conference on Mathematics, Vol.II, (Portuguese), Braga,1987," Univ.Minho, Braga, (1987), 564 - 571.

[4] De,U.C. and Sarkar,A., On three-dimensional trans-Sasakian manifolds, Extracta Mathematicae,(2008),265 - 277.

[5] De, U.C.and Tripathi,M.M., Ricci tensor in 3-dimensional trans-Sasakian manifolds, Kyungpook Math.J.43(2)(2003),247 - 255.

[6] Janssen, D. and Vanhecke, L., Almost contact structures and curvature tensors, Kodai Math. J.,4(1981),1 - 27.

[7] Gill, H. and Dube, K.K., Generalized CR-Submanifolds of a trans Lorentzian para Sasakian manifold, Proc. Nat. Acad. Sci. India Sec. A Phys. 2(2006), $119-124$.

[8] Gray, A. and Hervella, L.M., The sixteen classes of almost Hermitian manifolds and their linear invariants, Ann. Math. Pura Appl., (4) 123 (1980), 35 - 58. 
[9] Ikawa, T. and Erdogan, M., Sasakian manifolds with Lorentzian metric, Kyungpook Math. J. 35(1996), $517-526$.

[10] Marrero, J.C., The local structure of trans-sasakian manifolds, Ann.Mat.Pura Appl.(4)162 (1992), $77-86$.

[11] Marrero, J.C. and Chinea D., On trans-sasakian manifolds, (Spanish), in "Proceedings of the XIVth Spanish-Portuguese Conference on Mathematics, Vol.I-III, (Spanish),Puerto de la Cruz,1989", Univ.La Laguna,La Laguna,1990, 655-659.

[12] Matsumoto, K., On Lorentzian paracontact manifolds, Bull. Yamagata Univ. Nat. Science, 2(1989), $151-156$.

[13] Matsumoto,K. and Mihai,I., On a certain transformation in a Lorentzian Para-Sasakian Manifold, Tensor (N.S.), 47(1988), $189-197$.

[14] Matsumoto, K. , Mihai,I. and Rosca, R., $\xi$-null geodesic gradient vector fields on a Lorentzian para-Sasakian manifold. J. Korean Math. Soc. 32(1995), $17-31$.

[15] Mihai, I., Oiaga, A. and Rosca, R., Lorentzian Kenmotsu manifolds having two skew- symmetric conformal vector fields, Bull. Math. Soc. Sci. Math. Roumania, 42(1999), 237 - 251.

[16] Mihai,I. and Rosca, R., On Lorentzian P-Sasakian Manifolds, Classical Analysis. World Scientific, Singapore, $1992155-169$.

[17] Oubina, J.A., New classes of almost contact metric structures, Publicationes Mathematicae Debrecen 32 (1985), $21-38$.

[18] Shaikh, A. A., Baishya, K. K. and Eyasmin, S., On D-homothetic deformation of transsasakian structure, Demonstratio Mathematica, 1 2008, 171 - 188.

[19] Takahashi, T., Sasakian $\phi$-symmetric spaces, Tohoku Math.J.(2)29, (1)(1977),91-113.

[20] Yaliniz, A.F., Yildiz, A. and Turan, M., On three-dimensional Lorentzian $\beta$ - Kenmotsu manifolds, Kuwait J. Sci. Eng. 36 (2009), $51-62$.

[21] Yildiz, A., Turan, M. and Murathan, C., A class of Lorentzian $\alpha$ - Sasakian manifolds, Kyungpook Math. J. 49(2009), 789 - 799.

Current address: Uday Chand De,, Department of Pure Mathematics,, Calcutta University ,, 35 Ballygunge Circular Road, Kol 700019, West Bengal, INDIA. , Krishnendu De, Konnagar High School(H.S.),, 68 G.T. Road (West),Konnagar,Hooghly, Pin.712235, West Bengal, INDIA.

E-mail address: uc_de@yahoo.com, krishnendu_de@yahoo.com

$U R L$ : http://communications. science. ankara.edu.tr/index. php?series=A1 